\title{
Behavioural Artificial Intelligence: An Agenda for Systematic Empirical Studies of Artificial Inference
}

\author{
Tore Pedersen $^{1,3}$ and Christian Johansen ${ }^{2}$
}

\author{
${ }^{1}$ Center for Intelligence Studies, Norwegian Defence Intelligence School, Oslo \\ ${ }^{2}$ Department of Technology Systems, University of Oslo \\ ${ }^{3}$ Department of Psychology, Bjørknes University College, Oslo
}

Tore Pedersen (corresponding author)

tore.pedersen@feh.mil.no

https://orcid.org/0000-0002-4502-0772

Christian Johansen

cristi@ifi.uio.no

https://orcid.org/0000-0002-1525-0307

Tore Pedersen was partially supported by the project Oslo Analytics funded by the IKTPLUSS program of the Norwegian Research Council. Christian Johansen was partially supported by the project IoTSec - Security in IoT for Smart Grids, with Number 248113/O70 part of the IKTPLUSS program funded by the Norwegian Research Council.

About the authors:

Tore Pedersen is Associate Professor, Dean of Academic Studies, and Director of Center for Intelligence Studies (CIS) at the Norwegian Defence Intelligence School (NORDIS). He is also affiliated Associate Professor at the Department of Psychology, Bjorknes University College and Visiting Cognitive Scientist at the Department of Informatics, University of Oslo. Tore Pedersen received his $\mathrm{PhD}$ in Psychological Science from Karlstad University and he is currently engaged in research on Human - Technology Interaction, and cognitive aspects of National Intelligence and Security.

Christian Johansen is a researcher in the Precise Modelling and Analysis group of the University of Oslo (UiO) and member of the ConSeRNS interdisciplinary group Concurrent Security and Resilience for Networked Systems. He received $\mathrm{PhD}$ in 2010 from $\mathrm{UiO}$ and has worked since in different areas of computer science, including modelling of security protocols; modelling languages and verification of complex systems; models and tools for parallel programming and concurrent systems; (legal) electronic contracts. His work, more than 30 international articles, has been published in highly ranked conferences s.a. CONCUR, ATVA, or LFCS, and top journals s.a. JLAMP.

\begin{abstract}
Artificial Intelligence (AI) receives attention in media as well as in academe and business. In media coverage and reporting, $\mathrm{AI}$ is predominantly described in contrasted terms, either as the ultimate solution to all human problems or the ultimate threat to all human existence. In academe, the focus of computer scientists is on developing systems that function, whereas philosophy scholars theorize about the implications of this functionality for human life. In the interface between technology and philosophy there is, however, one imperative aspect of AI yet to be articulated: How do intelligent systems make inferences? We use the overarching concept “Artificial Intelligent Behaviour” which would include both cognition/processing and judgment/behaviour. We argue that due to the complexity and opacity of Artificial Inference, one needs to initiate systematic empirical studies of artificial intelligent behavior similar to what has previously been done to study human cognition, judgment and decision making. This will provide valid knowledge, outside of what current computer
\end{abstract}


science methods can offer, about the judgments and decisions made by intelligent systems. Moreover, outside academe - in the public as well as the private sector - expertise in epistemology, critical thinking and reasoning are crucial to ensure human oversight of the artificial intelligent judgments and decisions that are made, because only competent human insight into AI-inference processes will ensure accountability. Such insights require systematic studies of AI-behaviour founded on the natural sciences and philosophy, as well as the employment of methodologies from the cognitive and behavioral sciences.

Keywords: artificial intelligence; artificial inference; behavioral artificial intelligence; artificial intelligent behaviour; bias; transparency; accountability; ethics

\section{Introduction}

Artificial Intelligence (AI) can contribute significantly to the expansion of human judgment and decision making: AI learns and processes, while making inferences and drawing conclusions, similarly to what humans do, although with unsurpassed speed and capacity for handling complexity and volume. The strongest AI-algorithms are in fact developed based on knowledge about how the human brain functions, and insights into AI-processes are limited by the same characteristical constraints that limit insights into the human brain:

- In the same way as humans may have difficulties in accounting for how they have processed the information that has lead them to make a judgment, $\mathrm{AI}$ is characterized by having the same type of "difficulties", in that advanced AI-algorithms are not immediately accessible and comprehensible, not even for the experts that programmed them (Dahl 2018). The strongest AI-algorithms have already earned the status of being "black boxes", ${ }^{1}$ in very much the same way as the processes of the human brain are inaccessible for oversight and comprehension. The reason is simply the enormous complexity of the AI machinery.

- Inaccessibility to oversight of complex AI-algorithms inevitably leads to lack of accountability, which is an imperative prerequisite for citizens' trust in parliament, public authorities, the justice system, or to trust that private enterprises conform to regulations or contracts. Accountability is a term that comprises important aspects such as provenance of, access to, transparency of, and auditability of, algorithms and data. All these are important also for the reliability and validity of AI inference processes, which is a prerequisite for the assessments of AI accountability. But accountability is not studied sufficiently within the domain of Artificial Intelligence (Wachter, Mittelstadt, Floridi, 2017) even though concerns about the relation between accountability and bias was raised almost 30 years ago (Dix, 1992).

The general public has low awareness of how even simple technology is being used in democratic processes, policy- and decision-making. With an increased complexity in technology as a result of artificial intelligence, one is at risk of making judgments and decisions that do not conform to the requirements of accountability (Danaher 2016). When facing a technological complexity that is difficult or even impossible to comprehend, this may give rise to feeling disempowered. The satirical comedy "Little Britain" and the scene where "the computer says no"2 may also be interpreted as showing how humans become enslaved when facing the "computer's" sovereign and opaque autonomy.

1 Knight, Will (14 March 2017). "DARPA is funding projects that will try to open up AI's black boxes". MIT Technology Review. https://www.technologyreview.com/s/603795/the-us-military-wants-its-autonomousmachines-to-explain-themselves/

2 Sample, Ian (5 November 2017). "Computer says no: why making AIs fair, accountable and transparent is crucial". the Guardian. https://www.theguardian.com/science/2017/nov/05/computer-says-no-why-making-aisfair-accountable-and-transparent-is-crucial 
A new agenda. The technological development within the domain of $\mathrm{AI}$ is predominantly fueled by needs for functionality and cost-efficiency, while other aspects, such as safety and security, ethics, transparency, accountability and judicial aspects, have not attracted the same interest, although these aspects have had much attention from theorists within arts and humanities, most predominantly stated as philosophical concerns about the possible negative implications of AI on humans and society (e.g., Binns, 2018; Bostrom, 2016; Dahl, 2018; De Laat, 2018; Pagallo, 2018). Even if many have articulated why we need to gain more knowledge about $\mathrm{AI}$ and its opaque processes, no one has yet disclosed the link between AI-behaviour and AI-inference by describing how to study AI Behaviour. We argue in this paper that, even if complete transparency of AI processes is not achieved (Miller, Howe, Sonenberg, 2017; Samek, Wiegand, Müller, 2017; Biran and Cotton, 2017; Doshi-Velez and Kim 2017; Murdoch et al., 2019), for similar reasons as human cognitive processes are not immediately accessible from the 'outside', another type of transparency can nevertheless be achieved. By means of systematic studies of AI Behaviour, one could provide insightful evidence for important aspects in AI, such as accountability and soundness of inference.

We will parallel later in the paper our proposed Artificial Intelligence Behavioural studies with Tversky and Kahneman's (1974) studies of human cognition and behaviour (and of the many ways in which people systematically deviate from a rational agent behaviour) which ultimately lead to novel insights into, until then, similarly 'opaque' cognitive processes. Eventually, this insight lead to the discovery of the relationship between heuristic processing mechanisms and biased judgments. Our starting point is the perspective of the theorists that have articulated the need for more knowledge about why one needs more insight into important aspects of AI output. We extend this perspective, elaborating on the why by comparing human cognition and learning to algorithmic processing and AI learning, and by introducing the concept of AI inference and AI behaviour to the discussion. As a result, we propose a novel agenda for how the theorists' articulated why can be achieved.

Consequently, there are two imperative aspects that are not yet well articulated:

1. The importance of studying artificial intelligent behavior and artificial inference systematically by means of empirical studies and experimental methods from behavioural and cognitive sciences, and

2. The need for broad scientific competence in order to assess whether or not artificial intelligent judgments and decisions are reliable, valid and inferentially sound.

The first aspect regards the need for research on AI-behavior and inference as a consequence of the artificial intelligence's autonomous and self-learning algorithms being opaque and thus inaccessible to human oversight and control of the validity of the output. The second perspective regards accountability in public and private affairs due to the fact that artificial intelligent judgments and decisions must ultimately be regarded as a support for human or institutional judgments and decisions. These aspects require a broad competence derived from several scientific disciplines, researching how artificial intelligent behavior can be studied systematically, as well as whether or not the artificial intelligent behavior conforms to established principles of reliability and validity in inferences. Soundness of inference and absence of bias - or at least awareness and reduction of bias is a prerequisite for how much the processed knowledge can be trusted.

We thus propose a joint research endeavour - comprising both philosophical theorists from the arts and humanities, technology-developers from computer science, and empiricists from the social sciences, such as for example cognitive- and behavioral scientists - called Behavioral Artificial Intelligence, which would incorporate these perspectives. Behavioral Artificial Intelligence (BAI) would study the artificial inferences inherent in, and the manifested behaviour of, artificial intelligent systems in the same way as the social sciences have studied human cognition, inference and behaviour. We follow the traditions from other hybrid disciplines such as Behavioral Economics (e.g., Kahneman and Thaler, 2006), Behavioral Transportation Research (e.g., Pedersen, Friman and Kristensson, 2011; Gärling, Ettema and Friman, 2014) and Behavioural Computer Science (Pedersen, Johansen and Jøsang, 2018). The key aspect in these disciplines is that they complement and update 
traditional fields such as economics, transportation research and computer science, by providing (descriptive) empirical data about actual human cognition and behaviour from the cognitive sciences and the behavioral sciences instead of relying on assumptions about (prescriptive) rational behaviour.

Of particular importance in BAI are:

1. the relation - similarities and differences - between human cognition and algorithmic processing;

2. the relation between human learning and algorithmic (machine) learning; and

3. the process of inferring knowledge from data, thus arriving at valid and reliable judgments, made by an artificial intelligence system compared to how humans make judgements.

In the following sections we first elaborate on human cognition, learning and behaviour, and show how these are related to machine processing and output. Then we link reasoning and inference to important philosophical concepts, and show how human and machine inference must conform to sound principles of knowledge-generation, By thus disclosing the link between (AI-)inference and (AI-)behaviour we show that insights into AI-inference must be achieved with systematic studies of AI-behaviour, in the same way as insight into human inference have been achieved with systematic studies of human behaviour.

\section{Human Cognition and Artificial Intelligence Processes}

Artificial intelligence is now widely covered in the media, generating feelings ranging from fascination to fear (LeCun, Bengio, Hinton, 2015; Jordan and Mitchell, 2015; Parkes and Wellman, 2015; Boden 2016; Biamonte et al. 2017; Frégnac 2017; Ramprasad et al. 2017; Brundage et al., 2018). But what is artificial intelligence? A brief glance into the history of AI shows that Alan Turing, in his endeavour to construct "the mechanical brain" in the 1950s, was a prominent actor in the early development of artificial intelligence. Later developments included the works of the 1978recipient of the Nobel Prize in Economics, Herbert A. Simon (Newell, Shaw and Simon 1958; Newell and Simon 1972; Newell and Simon 1976; Simon 1979; Simon 1996) as well as the 1971-recipient of the ACM Turing Award, John McCarthy (McCarthy 1987; McCarthy and Hayes 1969). Although early AI-endeavours were predominantly in the symbolic tradition, based on a rational actor paradigm including forms of logic and reasoning systems, whereas later endeavours are non-symbolic and more influenced by empirical neuroscience (cfr Dix, 2016) ${ }^{3}$, their works nevertheless show that a significant part of the processes in AI are inspired by contemporary-times' knowledge about human cognition and how the human brain functions.

AI and machine learning methods like neural networks and reinforcement learning (Sutton and Barto, 2018) are examples of current algorithms directly inspired by human cognition, whereas evolutionary computation and genetic programming (Holland 1992; Bäck, Fogel, Michalewicz, 1997; Mitchell 1998; Coello, Lamont, Van Veldhuizen, 2007) are inspired from human biology and evolution. Whereas the symbolic AI-tradition is related to the cognitive System 2 and thus based on prescriptive rational agency, the non-symbolic empirical tradition is related to the cognitive System 1 and thus more descriptive, based on empirical evidence for (also non-rational) behaviour. One of the most popular concepts today is deep neural networks (DNN) (e.g., Demuth et al., 2014), which is even more similar to how the human brain functions. In DNN, artificial neurons are connected in a network where each single neuron communicates with a great number of other neurons, in very much the same way as the neurons in the human brain do. The network consists of several layers of neurons. One "input" layer is responsible for taking in a problem instance, like an image, and one "output" layer is responsible for producing the response, like yes/no whether a cat is present in the image. Several other "hidden" layers of neurons are involved in the processing, hence the concept "deep" and each single neuron encodes a specific aspect of the "problem" the DNN is designed for. In our problem of

3 The EPSRC 'Human-Like Computing' initiative aims to bridge this 'gap' between 'symbolic'/'rational' and 'neural'/'empirical’ AI. See: http://hlc.doc.ic.ac.uk/ 
recognizing cats in images some neuron will encode the fact that cats have a visible tail (or something even more seemingly insignificant than that). AI techniques are generally built in two stages: first $a$ learning stage where the DNN is trained on a dataset where the correct response is known for every input; and then an operational stage where the algorithm is used in the wild; given a new input it produces a decision or action (like changing the picture, or logging some inferred information about the picture). Sometimes these stages are combined, and an algorithm constantly learns if it has a mechanism to obtain some form of feedback on the responses that it produces. Even though there are differences between DNNs and the human brain, for example that DNNs are dependent on massive volumes of data in order to learn, whereas the human brain may be able to learn from being exposed to only one single instance of a phenomenon (i.e., data) ${ }^{4}$, the similarities are nevertheless striking and for both, the learning processes will always result in some type of behavioural output.

Simple algorithms vs artificial intelligence. Machines can be "simple", in the way that they are given predefined instructions - that is, algorithms - to perform a task and never deviate from these instructions when processing data, e.g. when locating specific information in a dataset or making a judgment about some attributes of the dataset (Minsky 1967; Knuth 1973; Dennett 1995). In simple machines the processing is transparent in the sense that it is possible, at least to some extent ${ }^{5}$, for humans both to understand the process and to have access to what the machine actually does with the data it processes. Algorithms when executed by a machine can be seen as the "behavior" of the machine (e.g., the returned results, the commands sent to some actuators, etc.) terminology, which appears in various specific areas of computer science research (Hennessy 1988; Bergstra, Ponse, Smolka, 2001; Hennessy and Rathke, 2004; Ancona et al., 2016; ). However, Artificial Intelligence (Russell and Norvig, 2016) is an example of a "complex" machine which is programmed (with algorithms) in a way that allows it to interact with its surroundings (in the form of data continuously fed in) and as a result of this interaction is allowed to make new algorithms and adjust existing algorithms after having "learned something new" from the surroundings. The adjusted internal execution of AI thus can be seen as adapted new behavior that AI learns continuously. Whereas the processes in a simple machine are transparent, the processes in intelligent systems are, as a consequence, opaque and thus not immediately accessible to human oversight, supervision and audit (Russell 1997; Rahwan and Simari, 2009).

In order to to have an intuition about the AI learning process, consider for example how the brain of a child develops through learning, beginning with birth and continuing by interaction with other humans as well as other parts of the child's surrounding environment. The child's brain is being "wired" in a particular way as a result of learning through such interactions, which we call experience. This can be compared to the way artificial intelligent systems functions: during the learning phase the neural network will be "wired" in a particular way as a result of interacting with the training data thus, the training data represents the artificial intelligent system's experiences. But what will happen if one allows an artificial intelligent network unlimited possibilities to learn through unlimited interaction with voluminous data sets over time, and moreover, the network is allowed to autonomously develop its "deep” neural network outside of human control (something which already is done by significant AI-actors like IBM and MIT)? What will such an artificial intelligent system be like, compared to the human brain? Will the AI system develop a personality, abilities, recognizable traits? What sorts of preferences will the system have and what types of inference-criteria will be the basis for its generation of valid and reliable knowledge? Will it always make objective, accurate and "just" judgments, or could it be biased in the same way as a human can be biased? In other words: how does AI infer and what behaviour does it exhibit?

To understand how artificial intelligent systems make judgments and provide decision-support for human and institutional decision-making in the context of identifiable accountability, one should adapt methodologies from empirical studies on human personality as well as on human judgment and

4 Cfr stimulus - response and classical conditioning

5 Even 'simple' codes can sometimes be difficult to fully comprehend, e.g., probabilistic programs (Gordon et al., 2014; Katoen et al., 2015) or concurrent programs (Andrews and Schneider, 1983; Ben-Ari, 2006; Dijkstra, 1965). 
decision making, from the cognitive sciences and the social and behavioral sciences. This would imply using cross-sectional, longitudinal or experimental designs. Cross-sectional designs would allow to compare a broad range of AI systems to each other with regard to characteristics like 'personality and preference' and the validity and reliability of AI 'judgments'. Longitudinal designs would allow us to monitor a single AI system's 'personality' and 'judgment' over time, providing information about possible fluctuations. Experimental studies would allow manipulations to help detect whether some types of input (or interactions) would affect 'personality' and 'judgment' more than others. True experimental design (also known as randomized controlled trials: RCT) would even allow the study of potential biases in AI systems as well as the causes of the biases (i.e., by means of manipulations), something which would provide valid and reliable answers to the concerns raised about machine learning biases, both by academe (Baeza-Yates, 2018; Crabtree et al., 2019; Dix, 1992; Dix 2018; Dwork 2011; Monroe, 2018; Zemel et al., 2013;) and regulatory authorities (USACM $2017)^{6,7}$. These concerns are explicitly raised, but yet no reliable answers have been provided, something which strongly signals the need for scientists to respond to the concern by exploring methodology in novel ways to arrive at the answers.

Extensions of Man. McLuhan describes in Understanding Media; The Extensions of Man (McLuhan, 1964) the difference between information per se and the specific medium in which the informational content is transmitted, e.g., in writing, orally, visually, tactile. An important point made by McLuhan is that a medium is an extension of human traits and behaviour, and thus an extension of humans as such, e.g.: the written word is an extension of thought and speech; tools and utensils are extensions of fingers and arms, clothing is an extension of the skin. Nowadays, many types of technology can be seen as even more advanced extensions of humans. We can easily perceive artificial intelligence as an extension of human cognition, memory - and inference.

Another aspect in McLuhan's analysis is that each and every medium has an inherent tendency to shift human attention away from the informational content that the medium is a vehicle for, and over to the medium itself, something which leads to the perception of the medium as the actual informational content, instead of being a vehicle for transmitting and communicating the informational content. Today, AI and the opacity of its "black box" processes may, in the same way as McLuhan perceived traditional media, easily become a medium that draws attention away from the judgments it actually carries out.

Machine learning algorithms (which we interchangeably call AI in this paper) are particularly well suited to quickly elicit correct information (probabilistically, i.e., to some degree of error) from a voluminous dataset, also if the data consists of information that is not structured. Natural language, or images, as opposed to data that is presented as numbers in rows and columns, are considered unstructured data. IBM Watson is an example of a machine capable of finding a correct answer from a voluminous dataset, provided that there actually exists a correct answer (Ferrucci, 2011; Ferrucci et al., 2013). IBM Watson cannot, however, look for clues to the answers "outside" the recorded datasets in the same ways as humans can - something which of course is a limiting factor for these types of intelligent systems. Because a machine such as IBM Watson can process voluminous information very fast, as opposed to a human who has limited capacity for processing and who is also considerably slower, the machine is an invaluable helper when large amounts of information need fast processing.

In Technopoly: The Surrender of Culture to Technology Neil Postman (Postman, 1992) made an analysis of technology that in many ways resemble McLuhan's thoughts: all types of traditional tools and technologies have inherent traits that make them particularly good at carrying out specific tasks,

6 New York City Council (2018). A Local Law in relation to automated decision systems used by agencies. http://legistar.council.nyc.gov/LegislationDetail.aspx?ID=3137815\&GUID=437A6A6D-62E1-47E2-9C42461253F9C6D0

7 EU Parliament (2016). EU Framework on algorithmic accountability and transparency. http://www.europarl.europa.eu/sides/getDoc.do?pubRef=-//EP//TEXT+WQ+E-2016$\underline{007674+0+\mathrm{DOC}+\mathrm{XML}+\mathrm{V} 0 / / \mathrm{EN}}$ 
such as the axe is good at cleaving, or the knife is good at cutting. Gibson (1979) adds momentum to McLuhan and Postman's analyses in a behavioural sense by showing that external objects are perceived by humans not only in the sense of having a particular shape or being placed in spatial distances from each other, but also by their 'affordances'. According to Gibson's Affordance Theory, objects are perceived as having potential for action - that is, it is the human perception of objects that drives (or prompts) human action. Thus, if a knife is good at cutting, it is because humans "allow" the knife to exhibit its inherent traits by being vehicles for the knife's potential. In the same way advanced technologies also have inherent traits and tendencies which make them good at carrying out specific tasks. Thus, a tendency emerges which makes the various types of tools and technologies "promote" the particular types of tasks that each tool and each technology is good at performing. However, whereas the inherent traits of simple tools are easily decoded, the same traits are not easily decoded - nor even discovered - in advanced technology. Thereby we forget - or, more correctly, we do not understand - AI's inherent traits and accompanying tendencies to promote specific processes that lead to specific outcomes. For example, when AI learns by interacting with a limited dataset that represents only a fragment of the social world, inferences about the larger social world may be drawn from a non-representative dataset, much in the same way as humans are also prone to do if not 'corrected'. Thus, one specific implication of an AI 'trait' is that AI would exhibit the same biases as humans, only extremely more 'efficiently'. This could lead to a substantial increase in biased judgments as a result of the powerful AI extension of human erroneous reasoning. An example of this powerful effect, albeit from traditional technology that is more familiar, is the grading of high school exams and the subsequent acceptance or rejection of students into universities or into the labor force. Today, we accept this technology as 'a given' (or even 'a natural') expression of human knowledge and skills when, in fact, the most prominent feature of exam grading is administrative simplification with the aim of ranking and sorting humans for some other end-purpose and merely taking the grade as a face-value ‘token’ for knowledge.

Adapting research methods from the cognitive sciences and the behavioural sciences to artificial inference and behaviour would help gather empirical data that will provide increased knowledge and insight into whether AI systematically exhibit specific and recognizable traits and preferences in the same way as humans do, and whether AI exhibit systematic tendencies to "behave" in a certain way in terms of having a tendency to employ specific types of inferences or to produce specific types of output (e.g., fallacies in formal reasoning or whether judgments and decisions are biased due to data not being representative of the 'true' population). It would however be interesting to see if future AI would be able to avoid being 'blind' to its own biases - that is, if AI would be able to somehow 'transcend' the ubiquitous human tendency to find justifications for own biases.

Whereas the research traditions in the arts, the humanities, and the social sciences, most notably Philosophy, have been concerned with the implications of technology for human life and human activities, the technological research traditions themselves have been more occupied with developing new technology with the aims that technology should solve existing problems like replacing the human in the workplace. Thus, the most important aspect for the technological sciences is the development of functionality. As a consequence philosophy has been focusing on ethical aspects of technology and has been concerned with the potential threats to humanity posed by technology if humans lose their control over technology (Bostrom 2014). The "division” of these two research fields is an example of a compartmentalization when the fields would in fact benefit from being multidisciplinary. This is why it is necessary to embrace a more holistic approach to AI - an approach that incorporates both empirical evidence about both the technological development of AI, and implications for the individual and societal domains in which AI is employed, including actual artificial inference and artificial intelligent behaviour. Regardless of whether it is a human or a machine that observes, make judgments or inferences, and concludes - individually or in interaction with its environment - both humans and artificial intelligent systems must nevertheless adhere to the same principles when generating knowledge that is supposed to be valid and reliable. In other words, they must all build their inferences on sound reasoning and representative empirical data. 


\section{Artificial Intelligence and Cognitive Bias}

Two modes of thinking. The cognitive and behavioral sciences have through decades provided substantial empirical evidence on how humans think, make judgments, assess, choose, and make decisions. The empirical evidence has resulted in theories and models that show a distinction between two typical modes of cognitive processing - that is, thinking - labeled System 1 and System 2 (Kahneman, 2003; Sloman, 2002). Whereas System 1 is a non-deliberate and automatic mode of thinking that is carried out by our brain while we are not consciously aware that we are making a judgment or an assessment, System 2 is the deliberate and non-automatic mode of thinking that we carry out while being absolutely consciously aware that we are making a judgment or an assessment. Cognition carried out by System 1 is commonly labeled intuitive thinking, whereas cognition carried out by System 2 is labeled analytical thinking. When we make "formal" judgments and decisions in situations where we are aware of what we are doing, and informed in the sense that we have relevant information of high quality and sufficient time to make a judgment, we are carrying out a rational and controlled judgment of a case by means of System 2 .

In the context of the continuously-processing and always-learning artificial neural network, the human System 1 could be seen as paralleling the automatic and fast response that the network provides when a new problem needs to be solved, whereas the human System 2 could be seen as paralleling the AI's feedback loops and reinforcement learning that identifies when the answer is not correct, and recalibrates the network so that it is more likely to produce a more accurate judgment and thus a more correct solution to a future similar problem. However, this tentative analogy is not clear-cut, as there are also similarities between the human analytical System 2 and the traditional 'symbolic' AIendeavours. Another analogy is between the human analytical System 2 and the traditional symbolic AI endeavours based on logics, which we see coming back into fashion to explain AI (e.g., Bottou, 2014; Vardi, 2018).

Intuitive Thinking: Heuristics as Bias-generators. On the other hand, many judgments and decisions that we make in everyday situations are often carried out without us being consciously aware of the fact that we are making a judgment or a decision. This is an example of a normal cognitive processing mode that is appropriate in many everyday situations. Our brain automatically filters out stimuli that are not relevant to the particular judgment that we are going to make right now. The process is going on without our conscious awareness, and it usually functions well. As seen from an evolutionary perspective, this intuitive reasoning-mode was highly appropriate in a less complex environment than ours; today, however, heuristics may lead to a biased output under conditions (and in environments) that are characterized by uncertainty. Thus, the appropriateness of the reasoningmode depends on the context: when we intuitively possess the knowledge and the skills needed to arrive at a correct judgment, fast heuristic reasoning works well. However, our brain has a tendency to automatically activate System 1 also in situations where we should instead have activated System 2 (e.g., in situations where we have too little knowledge or too little experience). This can easily lead to an incorrect judgment, usually called a cognitive bias (Tversky and Kahneman, 1974), caused by psychological mechanisms that are activated when we make judgments under uncertain conditions (e.g., when information is incomplete or too complex, or when the time-frame at our disposal is limited). These psychological mechanisms are labeled heuristics and they function as mental shortcuts activated without conscious awareness (Gilovich, Griffin and Kahneman, 2002; Tversky and Kahneman, 1974).

We mention two widespread heuristics (Kahneman, 2003):

- The accessibility/availability heuristic (Tversky and Kahneman, 1973) prompts us to infer (non-consciously) that information easily accessible in memory represents something that is also common or frequently occurring. For example, since it is easier to recall words beginning with the letter $\mathrm{R}$ than words where $\mathrm{R}$ is the third letter, people may wrongfully infer that words beginning with $\mathrm{R}$ are more frequent, just because the former 
is easier to recall (Tversky and Kahneman, 1974). Similarly, we may make a biased judgment about a phenomenon simply because there is high availability only to some instances in memory and not to others.

- The representativeness heuristic (Kahneman and Tversky, 1972) prompts us to make an instant judgment of an unfamiliar phenomenon based on a perceived similarity to a familiar phenomenon, even if this similarity is only superficial. For example, the colour of someone's skin, the way they dress, or the way they talk, are characteristics that, when they occur in combination with demographic features such as age, gender, or occupation, may prompt us to infer that specific individual characteristics and specific demographic features are strongly related. Thus, we may make a biased judgment about a person simply because we make an incorrect categorization due to an 'overactive' System 1.

If we are given feedback on a judgment we make, we will probably experience learning (although learning may also occur by association as a result of classical conditioning), and a future judgment may become more correct. But if System 1 does not receive feedback, or receives false feedback (e.g., not sufficiently, or wrongly "calibrated" to reality) we will not learn that the judgment is incorrect, and the incorrect judgment may sustain and be applied also in future situations. Interestingly, reinforcement learning in AI uses the same feedback methods (Sutton and Barto, 2018). The science of human thinking and learning have long traditions and convincing empirical evidence from longitudinal as well as experimental studies, both controlled in laboratory and taking place as naturalistic studies in the field. We argue that one must carry out empirical studies also of artificial inference and learning.

The two modes of thinking are similar to the way we learn. For example, children are given little or no formal education when they are learning their native language. Moreover, they are not consciously aware that they are learning a language. Thus, they learn the language intuitively, continuously interacting with their surroundings. As Burling (2005) notes, pertaining to learning, the most interesting part of language lies in the interplay between the production and the reception of language. This mode of learning has much in common with intuitive thinking. In the same way we learn as adults to make judgements about the world surrounding us and the everyday-situations. This intuitive learning process - also labeled implicit learning without conscious awareness (Frensch \& Runger, 2003) - is strongly related to intuitive judgments prompted by System 1. On the other hand, we may of course be consciously aware that we are learning and that we are in fact taking part in a learning process. When adults sign up for a course to learn a foreign language they are consciously aware of the learning. This learning process has much in common with the judgments we make when we are employing analytical thinking in System 2. In addition to having conscious awareness of the process, we will also receive explicit feedback about whether we have learned what we have been expected to learn. Thus, we learn to use the foreign language correctly and we avoid making mistakes.

Human Aspects in the Machine. It has generally been assumed that a machine is objective and infallible compared to humans, but this has been widely questioned already since Alan Turing, and more recently also in legislations (EU, 2017; New York City Council, 2018; ACM US Public Policy Council, 2017). Most notably, a serious concern was raised in 2018, in a paper jointly signed by authors from prominent US and UK universities and research institutions, about the malicious use of artificial intelligence (Brundage at al., 2018). The common concern of these initiatives is the discovery that intelligent algorithms make incorrect judgments, something which has been known to lead to unethical and "unfair" decisions. For example, algorithms discriminate people (e.g., based on ethnicity, gender, or age) in automated decision-processes, such as in financial matters, in medical diagnostics, and in law enforcement.

- One explanation to why such incorrect judgments are made is that an algorithm is always initially programmed by a human; it is always a programmer that creates the initial algorithm that the machine uses when carrying out its tasks. A human's incorrect judgments (biases) may thereby be transferred to the machine algorithm, something which implies that 
the machine may exhibit the same incorrect judgments (biases) as the human that programmed it would have done.

- Another explanation, believed to be prevalent, is that the machine learns about the problem from a limited data set that does not necessarily represent all the aspects relevant for the problem. To put it another way, the algorithm learns from a limited sample that does not represent the larger population (i.e., the data is biased).

- Moreover, even if the machine makes a judgment that is correct in a formal and logical sense, the same judgment may be deemed inappropriate or unacceptable in an ethical sense, depending on the context; even if generalizing from demographic characteristics to individuals may be appropriate when screening for disease in order to provide health-care, it is unacceptable to reject job-applicants based on the same generalizations.

Thus, both the transfer of biases from programmers to machines, and machine-generated biases, imply that machines are not necessarily more infallible than humans, even if they are substantially faster and can process larger volumes of data. In fact, both humans and algorithms are fallible, but must nevertheless do their best to conform to principles of logic and reason and sound inference, when imposing a judgment on something in the social world and when learning something from the social world.

The systematic study of AI-bias. The aim of experimental-empirical behavioural research in the domain of psychology - more specifically, judgment, decision making, and inference - is to study whether humans exhibit general and universal tendencies to think, make judgments, decide, choose or behave in a certain way. For example, Tversky \& Kahneman, as well as contemporary and recent researchers, studied, at an aggregate (group) level, the individual human tendency to non-consciously activate heuristic thinking under conditions of uncertainty and thereby exhibit distinct types of biases in their judgments. If such tendencies is to be elicited in a scientifically valid way, one needs to select random samples of participants from a known population and carry out true experiments - that is, one or more variables need to be manipulated, the participants must be randomly assigned to either an experimental group or a control group, and all other conditions (except for the manipulated variable) must be identical for both groups. Analyses are then carried out using inferential statistics, either as regression or as analysis of variance, and results will show whether the sample at group level exhibits (or not) the hypothesized tendency. If the tendency exists, it is shown as a mean effect at group level. After establishing the overall effect at group level, the next step is to recruit new samples and analyse whether there are systematic individual differences in the tendency to exhibit the bias. For example, whether participants with some specific demographic characteristics (or specific personality characteristics, or specific cognitive "styles" of processing information), are more prone to exhibit the bias than others who do not share the same characteristics. Thus, this type of research starts with establishing the effect at group level and is pursued further by identifying differences at the individual level.

We argue that one needs to study AI-bias similarly. In order to systematically study AI-bias one must design and carry out empirical (preferably experimental) studies with AI-participants (i.e., systems or variations of their configurations or training data) in the same way as one has previously done with human participants. If one wants to gain knowledge about the potential general and universal tendency for AI to exhibit bias (both the biases that are already known, as well as hypothesized biases), one needs to study the phenomenon on an aggregate (group) level with a number of AIsystems as "participants". This way, one can detect a mean effect of the AI's tendency to exhibit a specific bias. A next step would be to look for "individual” differences at the individual AI-level, such as, whether AI-systems from one provider are more or less prone to exhibit a bias than AI-systems from another provider. There are now enough different AI systems for the same task, e.g. see the large AI competitions like Kaggle competitions ${ }^{8}$ or the traditional RoboCup events (Osawa et al. 1996).

8 https://www.kaggle.com/competitions 
Even from a single AI system one can create a group of multiple diverse AI systems on which to study bias by training the AI system on different training data sets. Even on the same data set an AI system can be configured in multiple ways, all of these forming the group that we want to study for biases.

\section{Inference -- Human and Artificial}

When crossing the border between the early traditional 'symbolic' AI-endeavours and the neuroscience-inspired 'DNNs' in later AI-endeavours - or when bridging this gap, such as in the initiative of the EPSRC Project 'Human-Like Computing' ${ }^{\text {- }}$ - important questions arise regarding future AI's ability to adhere to the philosophical underpinnings of reasoning. Would future AI understand the difference between different forms of 'being', and would it be able to distinguish between different ways of acquiring knowledge? Would future AI distinguish between different approaches to understanding 'universal' vs 'unique' realities, and would it distinguish between different types of 'truth'? These reasoning-related philosophical aspects that all need to be adhered to regardless of whether the agent is human or an AI-system, are all relevant to the study of AIbehaviour and the link between AI-inference and AI-behaviour.

Ways of knowing. In philosophy and metaphysics, two concepts are central to understanding what it means that something exists and how we can know something about that which exists. The concept regarding various forms of existence is known as ontology (Coffey, 1914) and the methods employed for acquiring knowledge about existence is known as epistemology (Coffey, 1917). As an example, our physical world can be understood as one type of existence, whereas our social world can be understood as another - different - type of existence. A physical object, say, a chair has one type of existence in the physical world, but may have another - or an additional - type of existence in the social world. In the physical world we are concerned about the chair's characteristics as a physical object, whereas in the social world, we are concerned about the meaning and significance of the chair - that is, the role that the chair has been given in the social world that it occupies.

If one is interested in distinguishing between different types of existence, one concerns oneself about ontology as a field of study. Being able to distinguish between various types of existence is valuable both in occupational life as well as in private life. There are different types of methods to acquire knowledge about the different types of existence. If one wants to know something about Santa Claus's physical existence as an entity actually living at the North Pole, this would require one type of investigative method (e.g., look for traces of Santa on places where he is claimed to live), whereas wanting to know something about Santa as a collective representation in children's minds would require a different type of investigative approach (e.g., interviews with children in order to gain knowledge about the characteristics of this collective representation) (Dylan et al., 2017). Different ontologies as well as the different epistemological approaches in each of the ontologies, can provide valuable knowledge, but it is nevertheless two different types of knowledge that we acquire with different methods that yield different meanings.

If one wants to make AI systems find out something about a social phenomenon, or to make a judgment or a decision about such a phenomenon, one should investigate which type of ontology the AI-system is employing and whether the AI-system's investigative methods are suited for acquiring knowledge about the phenomenon. Since metaphysics and philosophy of science (i.e., ontology and epistemology) is difficult for most humans to understand, how can we make sure that AI-systems will be able to handle these concepts and provide valid and reliable knowledge about complex phenomena in the social world? In the same way as humans are prone to make mistakes about the ontology and corresponding epistemology they base their knowledge on, AI output could also easily become biased if AI - or a human, when programming or feeding data to the AI - chooses the 'wrong' ontology or the 'wrong' epistemology. An example of this is Google Flu Trends (GFT) (Lazer et al., 2014), an intelligent system which was developed to predict outbreaks and spread of Influenza. GFT was initially fed with the officially recorded prevalence of Influenza (the condition is identified by

9 http://hlc.doc.ic.ac.uk/ 
physicians and then registered in national health databases) and simultaneously fueled with data from the Google Search Engine (where people typically search for symptoms related to Influenza). Due to being calibrated to both the official records and people's searches for symptoms, GFT could initially provide accurate predictions of the future outbreak and spread of Influenza. However, the accuracy of these initial predictions decreased; GFT began to predict a substantially higher rate of outbreak and spread of the condition than what was the actual outbreak and spread. This happened because GFT was not able to distinguish between people's search activities when they unknowingly had a common cold and were concerned about their health without actually having Influenza, and their search activities when they did in fact have Influenza. Since search activities when people are concerned about their health are quite similar to the search activities carried out when they do in fact have the condition, it is not possible, neither for machines nor for humans, to make accurate predictions based on search activity alone, because 'searching for Flu' and 'actual Flu' are two different ontologies.

The particular and the general. In addition to the existence of different types of methods for acquiring knowledge, it is also important to be clear about whether one wants to acquire knowledge about something that is specific, local and unique, or to acquire knowledge about something that is general, global and universal. The former is known as idiographic knowledge, whereas the latter is known as nomothetic knowledge (e.g., Robinson, 2011; cfr Hurlburt and Knapp, 2006). If one wants to acquire knowledge about a specific case one can conduct case-studies of the phenomenon or do indepth interviews with the person in which the phenomenon resides, or even ask other people about the phenomenon that resides in that specific person. If we are interested in gaining knowledge about how one specific person describes the driving forces of his or her life - that person's life-narrative - we will of course gain valuable knowledge about how this person understands his or her life and ascribes or interprets meaning into it. This type of investigative approaches - generally known to lead to idiographic knowledge - would provide valuable insight into a specific, local and unique phenomenon, but it does not warrant the generalization of the phenomenon to also be present in other persons when we have actually not studied other persons - that is, other specific cases. To ensure sound reasoning when making inferences about individual cases based on group statistics, the employment of System 2 - analytic thinking - is crucial.

On the other hand, if one wants to know something about whether a phenomenon - say, a personal trait, a preference, a taste, a tendency - is shared by many people, one should try to study the phenomenon broadly and make sure that the sample of the study consists of many people drawn from a larger population, so that the sample is representative. If one is interested in knowing, for example, if pre-school children prefer chocolate ice-cream over vanilla it is of course important to study a representative sample of pre-school children. One might find out that, in general, 60 percent of the girls prefer one flavor of ice-cream over the other and that boys are more concerned about eating an ice-cream and care less about whether it tastes chocolate or vanilla. This type of knowledge generally known as nomothetic knowledge - would provide valuable insight into the distribution of preferences in a larger group or a larger population, something that is important for many reasons, but it would not help with understanding whether a new and unknown member of that same population would share the same preference or exhibit the same tendency. The knowledge is valuable for understanding tendencies on group level, but does not automatically help us know whether we should serve chocolate ice-cream or vanilla ice-cream when a colleague is accompanied by her daughter to our office.

Now, let us ask ourselves: how would an AI-system go about to understand the meaning of a social phenomenon that exists in living people - such as a person's life-narrative - and how would an AIsystem go about to decide what it takes to ensure that a sample is representative of a larger population? Is it even possible that an AI-system would be able to relate to concepts such as idiographic and nomothetic knowledge? The only way to answer these questions is to study AI judgment, inference and behaviour in the same way as we have been studying human judgment, inference and behaviour. 
Three Modes of Inference. The questions posed in the previous paragraph is closely related to the act of making an inference. If one observes a specific phenomenon during similar occasions and under identical circumstances, one may rightfully claim that the phenomenon will occur also on the next occasion, provided the circumstances are identical. The term for this type of inference is inductive reasoning (e.g., Anderson, 1948; Bacon and Montagu, 1857). After many observations of the same phenomenon we make a rule about the phenomenon: it will occur again if the circumstances are the same as in previous observations. A key term in the previous sentence was many. How many consecutive observations does it take to be convinced that the phenomenon will occur again the next time? The answer is that it depends on several things, and that we can never be absolutely sure. Thus, probability is another key concept in inductive reasoning. In terms of meteorology, making a prediction that the gulf-stream - a part of the atlantic ocean current that brings warm water from the south to the north - will still be present tomorrow morning is a straightforward prediction to the extent that the gulf stream is a stable phenomenon. But in the case of non-stable phenomena or possible disruptions of stable phenomena, predictions are more difficult to make. Does the phenomenon currently seem to be somewhere on an upward trend? If so, we will often tend to extrapolate and predict that the ongoing trend will continue, although disruptions may occur and generate unforeseen surprises. To search for missing evidence and to know when induction might fail is difficult. Think for example of stock traders acting as if the current trend will continue forever.

How would an AI-system go about to determine how many observations it would take to engage in inductive reasoning and make a valid inference about the continuous future occurrence of a current phenomenon? Even if humans are fallible in making inductive inferences, an AI-system does not have the same opportunity as humans to be aware of data that is outside of 'reach' or to seek advice on this, so how would it keep its reasoning on track? How would AI understand that a disruptive event that is not directly related to the data could render further induction invalid? It seems that one needs to look for phenomena 'outside' the dataset, and even to look at other societal contexts, in order to infer whether further induction is appropriate or if it should be abandoned.

On the other hand, if you draw a conclusion - that is, if you infer - on the basis of a set of premises that are given (albeit not necessarily true) and thus are obligated to adhere to these premises, you are conducting reasoning strictly within the domain of formal logic. This type of reasoning is known as deductive reasoning (Kneale, 1945) and is different from inductive reasoning in that the premises do not need to be true in terms of corresponding to observations. Correct reasoning in this inferencemode follows from formal logic only, so it is entirely possible for AI-systems to adhere to this type of reasoning, given that the premises in the formal arguments are recognizable to the system. However, there are not many things in the social world of human affairs that fit easily into such a structure of formal logic. It is, of course, possible to code instances in the social world and fit them into the structure of formal logic, so that AI-systems may be able to help us to make valid inferences, particularly if there are many premises in a structure of argument. If so, AI-systems are much better than humans to process a large number of informational components. Thus, this reasoning mode probably represents the potential of traditional AI at its best, but it needs human coding of instances before the AI-processing and it needs human oversight of AI-output. It is questionable whether an AIsystem that only processes its inferences in deduction-mode can be called intelligent.

This difference between induction and deduction indicates that there are more than one way to understand the concept of truth - or to decide what it takes to call something true. One definition of truth is that a claim about, or a description of, something is true if the claim or the description corresponds with the "something" such as it actually is in the real world. If we make a claim about an instance in the real world, for example that a specific apple in a specific fruit bowl on a specific table is green, and can also observe that this specific apple is in fact green, then our claim or description is true. The essence of such a definition of truth is correspondence: if our claim corresponds with things as they actually are, then our claim is true. Thus, this definition is generally known as the correspondence principle of truth (e.g., Kirkham and Kirkham, 1992). The correspondence principle is closely related to inductive reasoning in that it presupposes - or at least favors - observations of instances so that they can be compared to what is claimed about them. 
For deductive reasoning, however, it makes less sense to talk in relation to truth-as-correspondence. Deductive reasoning does not relate to an "external" observable reality in the same way as inductive reasoning does, but rather to an "internal" relation between the premises of an argument - that is, between the premises and the conclusion. Thus, deductive reasoning does not rely on empirical, observable data, in order to be valid; it concerns itself only about the internal consistency and coherence between the premises and the conclusion in an argument. This is known as the coherence theory of truth, or truth-as-coherence (Rescher, 1973). If the whole argument is consistently coherent internally - regardless of whether the premises are "correspondence-true" or not - the argument is true in the sense that it is valid.

The value of the deductive inference mode and the coherence theory of truth is that it helps us reason in a way that follows formal logic. If we adhere to these principles, we can trust our reasoning more than if we do not adhere to them. Even if we have separated inductive reasoning from deductive reasoning, and even if we have separated truth-as-correspondence and truth-as-coherence, generating knowledge about the social world will more often than not employ both inductive and deductive reasoning. This process is generally known as the hypothetico deductive method (e.g., Popper, 1948), first proposed by Christiaan Huygens in the 17 th century ${ }^{10}$, in which one adheres to the principles of formal logic - to ensure consistency, coherence and sound reasoning - while at the same time observe whether the premises in the arguments in fact correspond with the same instances as they are observable or manifest in the external (social) world. Although science progresses more in the sense of shifting from 'old' to new paradigms when new evidence suggests that older paradigms must be discarded (Kuhn, 1962) than by continuous Popperian falsification, many types of empirical research are carried out by means of the hypothetico deductive method.

One could claim that modern AI-systems have adopted more the correspondence-principle of truth, employing more an inductive reasoning where their training data comprises the many observations about the problem at hand. Reinforcement-style of AI would constantly add more observations of true or false instances of the problem. However, AI-systems are programs, and as such employ formal logic in their code through the programming languages and algorithms, and more recently also through the mathematical theories behind. Thus we would say that AI, more than other traditional ITsystems which are only logic-based and deductive, combine the two truth-principles, and maybe adhere more to the Popperian approach than other systems. However, we feel that this combination has not been sufficiently recognized nor harnessed. We would thus propose more work in this direction, combined with understandings coming from the social sciences, e.g., on the lines drawn by the EPSRC Project 'Human-Like Computing' and aiming at offering AI systems abductive-reasoning abilities.

Abductive Reasoning. Many instances in the social world, however, do not lend themselves easily to observation as such. Think for example about the simple concepts of love, hate, or friendship. In order to solve the "problem" of unobservable instances, we usually operationalize them. Instead of observing love as such, we can instead look at cognitive or behavioral operationalizations of love: what do people think or say about the ones they love and how do people behave towards the ones they love? Thus, we can "observe" constructed concepts by observing the manifest operationalizations of the concepts.

Another way to solve the "problem" of non-observable or non-determinable instances in the social world is to employ rhetorics - that is, to determine the power or the reasonableness of arguments as they manifest in language. Since not all instances in the social world fit into the structure of formal logic (deductive reasoning) and since not all instances are readily observable (inductive reasoning) we are dependent on language to solve potential "problems" between people. The field of rhetorics acknowledges this "shortcoming" when it claims that formal-logical arguments are not always employable, whereas rhetorical arguments are more often employable. In rhetorics, formal-logical

10 https://www.britannica.com/science/hypothetico-deductive-method 
arguments (deductive reasoning) are known as syllogisms (e.g., Smiley, 1973), whereas rhetorical arguments are known as enthymemes (Jackson and Jacobs, 1980). Contrary to a syllogism, which is either true or false - and also "rare" in the real world of 'practical' problems - enthymemes are not true or false as such, but are instead more or less convincing as arguments. Since an enthymeme is an argument that does not have the consistency and coherence of strict formal logic, and thus cannot be determined as true or false, it is instead important to generate arguments that are as convincing as possible. Since we are not always able to collect evidence of, or verify, all the components (premises) in an argument, we need instead to "make up for" the discrepancy by making the best arguments that we can in the situation.

In the domain of inferences (deductive reasoning and inductive reasoning) this type of enthymatic arguments (as they are labeled in rhetorics) are related to abductive reasoning - that is, abductive reasoning (Walton, 2014) is a "do-your-best" type of inference that is employed when we find ourselves in a situation where we need to handle "syllogisms where one or more premises are missing”. In fact, most situations in the real world are like this.

Again, a question arises: How would AI solve formal-logically "wicked" real-life-problems and make up for missing premises in incomplete arguments? Would AI learn how to "make up for" missing premises in arguments and mimic the human way - even if not always sucessful - of "doing-its-best" by employing the rhetorical devices of abductive reasoning? Would future AI 'transcend' its 'traditional' formal-logic 'way of life' and enter into the domain of human-like reasoning when it infers and subsequently exhibits its behaviour?

\section{Conclusion}

Even if humans and machines are fundamentally different entities, they are nevertheless similar in one aspect: Both make judgments and inferences that support individual and institutional decisions and both take part in decision making. These decisions have consequences for individual humans as well as for society. It is therefore necessary to understand not only human inferences and human behaviour, but also Artificial Inferences and Artificial Intelligent Behaviour, so that we can verify the reliability, validity and accountability of the judgments and the decisions that artificial intelligent systems make. In this context it is necessary to mobilize a comprehensive, unified and multidisciplinary research agenda that includes the breadth of academic disciplines such as natural sciences and technology, humanities and liberal arts, and social sciences. One should not be content with developing artificial intelligent systems, focusing merely on the functionality of the systems. Neither should theoretical analysis be carried out detached from those who develop the intelligent systems. Instead, these two academic disciplines should collaborate and they should let themselves be inspired by the methods used in the cognitive sciences and the behavioural sciences to start studying artificial inference and artificial intelligent behaviour. Systematic empirical studies of artificial intelligent inference and behaviour is necessary to identify and obtain knowledge of AI's actual inference and behaviour (AI's judgments and decisions), as a means of obtaining accountability - or at least to identify the current state of accountability - in corporate and government decision making. Moreover, empirical studies of AI's ability to adhere to the specific context it operates within would be of great importance; formally correct reasoning (and judgment) in one context (e.g., screening for diseases) would not necessarily be acceptable in a different context (e.g., rejecting job-applicants) Without such a unified approach, McLuhan's and Postman's analyses of media and technology as autonomous extensions of man, could soon be applied on artificial intelligence, as an equivalent autonomous extension of man and society beyond oversight, control, accountability, and audit.

The agenda is, however, open as we need first to understand how proven methods of studying human judgment, behaviour and inference can be adapted and used on AI. This has not been done before (only formal methods from computer science are currently being tried out) and thus it represents a novel approach to unravel AI-opacity in the endeavour to provide transparency and accountability. In this joint enterprise, the key to disclosing the link between AI-inference and AI-behaviour is to 
employ the philosophical foundations of reasoning on the study of AI-behaviour in specific contexts that have specific purposes and lead to specific outcomes.

Acknowledgements: We are very grateful to the anonymous reviewers for their valuable comments that have improved this paper.

\section{References}

USACM: US Public Policy Council of The Association for Computing Machinery (2017). Statement on Algorithmic Transparency and Accountability. New York: Association for Computing Machinery.

Ancona, D., Bono, V., Bravetti, M., Campos, J., Castagna, G., Deniélou, P. M., ... \& Yoshida, N. (2016). Behavioral types in programming languages. Foundations and Trends in Programming Languages, 3(23), 95-230.

Anderson, F. H. (1948). The Philosophy of Francis Bacon. Chicago Illinois.

Andrews, G. R., \& Schneider, F. B. (1983). Concepts and notations for concurrent programming. ACM Computing Surveys (CSUR), 15(1), 3-43.

Bacon, F., \& Montagu, B. (1857). The Works of Francis Bacon (Vol. 1). Parry \& McMillan.

Baeza-Yates, R. (2018). Bias on the web. Communications of the ACM, 61(6), 54-61.

Bäck, T., Fogel, D. B., \& Michalewicz, Z. (1997). Handbook of evolutionary computation. CRC Press.

Ben-Ari, M. (2006). Principles of concurrent and distributed programming. Pearson Education.

Bergstra, J. A., Ponse, A., \& Smolka, S. A. (Eds.). (2001). Handbook of process algebra. Elsevier.

Biamonte, J., Wittek, P., Pancotti, N., Rebentrost, P., Wiebe, N., \& Lloyd, S. (2017). Quantum machine learning. Nature, 549(7671), 195.

Binns, R. (2018). Algorithmic Accountability and Public Reason. Philosophy \& Technology 31:543-556.

Biran, O. \& Cotton, C. (2017). Explanation and justification in machine learning: A survey. In IJCAI-17 workshop on explainable AI (XAI) (Vol. 8).

Boden, M. A. (2016). AI: Its nature and future. Oxford University Press.

Bostrom, N. (2014). Superintelligence: Paths, Dangers, Strategies. Oxford University Press.

Bottou, L. (2014). From machine learning to machine reasoning. Machine learning, 94(2), 133-149.

Brundage M, Avin S, Clark J, Toner H, Eckersley P, Garfinkel B, Dafoe A, Scharre P, Zeitzoff T, Filar B, Anderson $\mathrm{H}$ (2018). The malicious use of artificial intelligence: Forecasting, prevention, and mitigation. arXiv preprint arXiv:1802.07228.

Burling, R. (2005). The talking ape: How language evolved (Vol. 5). New York: Oxford University Press.

Coello, C. A. C., Lamont, G. B., \& Van Veldhuizen, D. A. (2007). Evolutionary algorithms for solving multiobjective problems, 2nd edition. Springer.

Coffey, P. (1914). Ontology, Or, The Theory of Being: An Introduction to General Metaphysics (Vol. 25). Longmans.

Coffey, P. (1917). Epistemology; Or, The Theory of Knowledge: An Introduction to General Metaphysics (Vol. 1). Longmans.

Crabtree, A., Urquhart, L. and Chen, J, Right to an Explanation Considered Harmful (April 8, 2019). Edinburgh School of Law Research Paper Forthcoming. Available at SSRN: https://ssrn.com/abstract=3384790 or http://dx.doi.org/10.2139/ssrn.3384790

Dahl, E. S. (2018). Appraising Black-Boxed Technology: the Positive Prospects. Philosophy \& Technology 31:571-591.

De Laat, P. B. (2018). Algorithmic Decision-Making Based on Machine Learning from Big Data: Can Transparency Restore Accountability? Philosophy \& Technology 31:525-541

Danaher, J. (2016). The threat of algocracy: reality, resistance and accommodation. Philosophy \& Technology, 29(3), 245-268.

Demuth, H. B., Beale, M. H., De Jess, O., \& Hagan, M. T. (2014). Neural network design. Martin Hagan.

Dennett, D. (1995). Darwin's Dangerous Idea: Evolution and the Meanings of Life. Simon \& Schuster.

Dix, A (1992). Human issues in the use of pattern recognition techniques. In: Neural Networks and Pattern Recognition in Human Computer Interaction. Eds. R. Beale and J. Finlay. Ellis Horwood. 429-451.

Dix, A. (2016). Human-Like Computing. (personal report on the EPRC workshop of the same name) http://alandix.com/blog/2016/02/23/human-like-computing/

Dix, A. (2018). Sufficient Reason. Keynote at HCD for Intelligent Environments, BHCI, Belfast, 3rd July 2018. http://alandix.com/academic/talks/sufficient-reason-2018/

Dijkstra, E. (1965). Solution of a Problem in Concurrent Programming Control. Communications of the ACM, 8(9), 569. 
Doshi-Velez, F., \& Kim, B. (2017). Towards a rigorous science of interpretable machine learning. arXiv preprint arXiv:1702.08608.

Dwork, C. (2011). A firm foundation for private data analysis. Communications of the ACM, 54(1), 86-95.

Dylan, H., Goodman, M. S., Jackson, P., Jansen, P. T., Maiolo, J., \& Pedersen, T. (2017). The way of the Norse Ravens: merging profession and academe in Norwegian national intelligence higher education. Intelligence and National Security, 1-17.

Ferrucci, D. (2011). How it all began and what's next. IBM Research. https://www.ibm.com/blogs/research/2011/12/dave-ferrucci-at-computer-history-museum-how-it-allbegan-and-whats-next/

Ferrucci, D., Levas, A., Bagchi, S., Gondek, D., og Mueller, E. T. (2013). Watson: beyond jeopardy!. Artificial Intelligence, 199, 93-105.

Frégnac, Y. (2017). Big data and the industrialization of neuroscience: A safe roadmap for understanding the brain?. Science, 358(6362), 470-477.

Frensch, P. A., \& Runger, D. (2003). Implicit Learning. Current Directions in Psychological Science, 12, pp 13-18.

Gibson, J. J. (1979). The theory of affordances. In: The Ecological Approach to visual perception. Boston: Houghton-Mifflin, 1979.

Gilovich, T., Griffin, D. \& Kahneman, D. (2002). Heuristics and Biases: The Psychology of Intuitive Judgment. United Kingdom, Cambridge: Cambridge University Press.

Gordon, A. D., Henzinger, T. A., Nori, A. V., \& Rajamani, S. K. (2014). Probabilistic programming. In Proceedings of the 36th International Conference on Software Engineering (ICSE) -- Future of Software Engineering track (pp. 167-181). ACM.

Gärling, T., Ettema, D., \& Friman, M. (Eds.). (2014). Handbook of sustainable travel. Springer.

Hennessy, M. (1988). Algebraic theory of processes. MIT press.

Hennessy, M., \& Rathke, J. (2004). Typed behavioural equivalences for processes in the presence of subtyping. Mathematical Structures in Computer Science, 14(5), 651-684.

Holland, J. H. (1992). Adaptation in natural and artificial systems: an introductory analysis with applications to biology, control, and artificial intelligence. MIT press.

Hurlburt, R. T., \& Knapp, T. J. (2006). Münsterberg in 1898, not Allport in 1937, introduced the terms 'idiographic' and 'nomothetic' to American psychology. Theory \& Psychology, 16(2), 287-293.

Jackson, S., \& Jacobs, S. (1980). Structure of conversational argument: Pragmatic bases for the enthymeme. Quarterly Journal of Speech, 66(3), 251-265.

Jordan, M. I., \& Mitchell, T. M. (2015). Machine learning: Trends, perspectives, and prospects. Science, 349(6245), 255-260.

Kahneman, D. (2003). A Perspective on Judgment and Choice: Mapping Bounded Rationality. American Psychologist, 58, pp 697-720.

Kahneman, D., \& Thaler, R. H. (2006). Anomalies: Utility maximization and experienced utility. The Journal of Economic Perspectives, 20(1), 221-234.

Kahneman, D., \& Tversky, A. (1972). Subjective probability: A judgment of representativeness. Cognitive psychology, 3(3), 430-454.

Katoen, J. P., Gretz, F., Jansen, N., Kaminski, B. L., \& Olmedo, F. (2015). Understanding probabilistic programs. In Correct System Design -- Symposium in Honor of Ernst-Rüdiger Olderog (Vol 9360 of Lecture Notes in Computer Science, pp. 15-32). Springer.

Kirkham, R. L., \& Kirkham, R. L. (1992). Theories of truth: A critical introduction (No. s 401). Cambridge, MA: MIT press.

Kneale, W. (1945, January). Truths of logic. In Proceedings of the Aristotelian Society (Vol. 46, pp. 207234). Aristotelian Society, Wiley.

Knuth, D. E. (1973). The Art of Computer Programming Vol.1: Fundamental Algorithms (2nd ed.). AddisonWesley Publishing.

Kuhn, T. S. (1962). The structure of scientific revolutions. Chicago and London.

Lazer, D., Kennedy, R., King, G., \& Vespignani, A. (2014). The parable of Google Flu: traps in big data analysis. Science, 343(6176), 1203-1205.

LeCun, Y., Bengio, Y., \& Hinton, G. (2015). Deep learning. Nature, 521(7553), 436.

McCarthy, J. (1987). Generality in artificial intelligence. Communications of the ACM, 30(12), 1030-1035.

McCarthy, J., \& Hayes, P. J. (1969). Some philosophical problems from the standpoint of artificial intelligence. Readings in artificial intelligence, 431-450.

McLuhan, M. (1964). Understanding Media: The Extensions of Man. USA: MIT Press.

Miller, T., Howe, P., \& Sonenberg, L. (2017). Explainable AI: Beware of inmates running the asylum or: How I learnt to stop worrying and love the social and behavioural sciences. arXiv preprint arXiv:1712.00547. 
Minsky, M. L. (1967). Computation: Finite and Infinite Machines. Englewood Cliffs: Prentice-Hall.

Mitchell, M. (1998). An introduction to genetic algorithms. MIT press.

Monroe, D (2018). “AI, explain yourself”. Communications of the ACM, (61)(11), Pages 111310.1145/3276742. https://cacm.acm.org/magazines/2018/11/232193-ai-explain-yourself/fulltext

Murdoch, W. J., Singh, C., Kumbier, K., Abbasi-Asl, R., \& Yu, B. (2019). Interpretable machine learning: definitions, methods, and applications. arXiv preprint arXiv:1901.04592.

Newell, A., Shaw, J. C., \& Simon, H. A. (1958). Elements of a theory of human problem solving. Psychological review, 65(3), 151. DOI:_http://psycnet.apa.org/doi/10.1037/h0048495

Newell, A., \& Simon, H. A. (1972). Human problem solving (Vol. 104, No. 9). Englewood Cliffs, NJ: Prentice-Hall.

Newell, A., \& Simon, H. A. (1976). Computer science as empirical inquiry: Symbols and search. Communications of the ACM, 19(3), 113-126.

Osawa, E., Kitano, H., Asada, M., Kuniyoshi, Y., \& Noda, I. (1996). RoboCup: The robot world cup initiative. In Proceedings of the Second International Conference on Multi-Agent Systems (ICMAS), Kyoto, Japan (pp. 9-13).

Pagallo, U. (2018). Algo-Rhythms and the Beat of the Legal Drum. Philosophy \& Technology 31:507-524

Parkes, D. C., \& Wellman, M. P. (2015). Economic reasoning and artificial intelligence. Science, 349(6245), 267-272.

Pedersen, T., Friman, M., \& Kristensson, P. (2011). Affective forecasting: predicting and experiencing satisfaction with public transportation. Journal of Applied Social Psychology, 41(8), 1926-1946.

Pedersen, T., Johansen, C., \& Jøsang, A. (2018). Behavioural Computer Science: an agenda for combining modelling of human and system behaviours. Human-centric Computing and Information Sciences, 8(1), 7.

Popper, K. R. (1948). Objective Knowledge: An Evolutionary Approach. Ox-43.

Postman, N. (1992). Technopoly: The Surrender of Culture to Technology: US, New York: Knopf, 1992.

Rahwan, I., \& Simari, G. R. (2009). Argumentation in artificial intelligence. Springer.

Ramprasad, R., Batra, R., Pilania, G., Mannodi-Kanakkithodi, A., \& Kim, C. (2017). Machine learning in materials informatics: recent applications and prospects. npj Computational Materials, 3(1), 54.

Rescher, N. (1973). The coherence theory of truth (pp. 54-64). Oxford: Clarendon Press.

Robinson, O. C. (2011). The idiographic/nomothetic dichotomy: Tracing historical origins of contemporary confusions. History and Philosophy of Psychology, 13(2), 32-39.

Russell, S. J. (1997). Rationality and intelligence. Artificial intelligence, 94(1-2), 57-77.

Russell, S. J., \& Norvig, P. (2016). Artificial intelligence: a modern approach. Pearson Education Limited.

Samek, W., Wiegand, T., \& Müller, K. R. (2017). Explainable artificial intelligence: Understanding, visualizing and interpreting deep learning models. arXiv preprint arXiv:1708.08296.

Simon, H. A. (1979). Rational decision making in business organizations (Nobel Prize lecture). The American economic review, 69(4), 493-513.

Simon, H. A. (1996). The sciences of the artificial. MIT press.

Sloman, S. A. (2002). Two systems of reasoning. I T. Gilovich, D. Griffin og D. Kahneman (red): Heuristics and Biases: The Psychology of Intuitive Judgment. United Kingdom, Cambridge: Cambridge University Press

Smiley, T. J. (1973). What is a Syllogism?. Journal of philosophical logic, 2(1), 136-154.

Sutton, R. S., \& Barto, A. G. (2018). Reinforcement learning: An introduction, 2nd edition. MIT press.

Tversky, A., \& Kahneman, D. (1973). Availability: A heuristic for judging frequency and probability. Cognitive psychology, 5(2), 207-232.

Tversky, A og Kahneman, D (1974): Judgment under uncertainty: Heuristics and Biases. Science, 185 (4157), 1124-1131.

Vardi, M. (2018). Machine learning and logic: Fast and slow thinking. Invited talk at the Summit on Machine Learning Meets Formal Methods. https://easychair.org/smart-program/FLoC2018/SoMLMFM-2018-07-13.html\#talk:76996

Walton, D. (2014). Abductive reasoning. University of Alabama Press.

Wachter, S., Mittelstadt, B., \& Floridi, L. (2017). Transparent, explainable, and accountable AI for robotics. Science Robotics, 2(6), eaan6080.

Zemel, R., Wu, Y., Swersky, K., Pitassi, T., \& Dwork, C. (2013). Learning fair representations. In International Conference on Machine Learning (pp. 325-333). 